\title{
MOTIVACIÓN, CONSUMO Y APRECIACIONES DE FACEBOOK POR PARTE DE JÓVENES UNIVERSITARIOS: EL CASO DE LA RED UCSC CHILE
}

\author{
KARINA LORETO CRESPO* \\ ÁlVARO ElguETa RUIZ** \\ ALEJANDRO RIFFO PARRA***
}

\begin{abstract}
RESUMEN
Facebook se perfila como el Sitio de Redes Sociales (SRS) más popular de Chile. Son miles los jóvenes que establecen una identidad en este portal con el fin de armar su propia red de contactos y, desde ahí, generan una socialización que afecta directamente tanto su intimidad como su vida socioafectiva.

La presente comunicación pretende describir y analizar las motivaciones, usos y consumo que los miembros de la red UCSC Chile realizan en Facebook.com desde una perspectiva cualitativa.

Las conclusiones del escrito sugieren que Facebook más que un espacio social, es un producto cultural cuyo consumo a nivel macro se ha convertido en moda. No obstante, la coproducción de contenidos ha tendido a la práctica de «prosumo», pues los usuarios son también productores en este ambiente que prioriza los lazos fuertes y débiles de las comunidades personales.
\end{abstract}

PALABRAS ClaVe: SITIOS DE REDES SOCIALES, FACEBOOK, PROSUMO

* Licenciada en Comunicación Social, Periodista Universidad Católica de la Santísima Concepción (UCSC), Concepción, Chile.

** Profesor y Periodista. Doctor en Comunicación Pública. Docente de la carrera de Sociología Universidad ARCIS Concepción.

E-Mail: aelguetar@gmail.com.

*** Licenciado en Comunicación Social, Periodista UCSC. 


\title{
MOTIVAÇÃO, CONSUMO \\ E AVALIAÇÃO DO FACEBOOK
}

\begin{abstract}
RESUMO
O Facebook surge como o Sítio de Redes Sociais (SRS) mais popular do Chile. São milhares os jovens que estabelecem uma identidade neste portal com o objetivo de construir sua própria rede de contatos e a partir daí, geram uma socialização que afeta diretamente tanto sua intimidade como sua vida socioafetiva. Esta comunicação pretende descrever e analisar as motivações, usos e consumo que os membros da rede UCSC Chile realizam no Facebook.com a partir de uma perspectiva qualitativa. As conclusões do artigo sugerem que o Facebook, mais que um espaço social, é um produto cultural cujo consumo em nível macro se transformou em moda. Mesmo assim, a co-produção de conteúdos apresenta tendência à prática do "prosumo», pois os usuários são também produtores neste ambiente que prioriza os laços fortes e fracos das comunidades pessoais.
\end{abstract}

PALAVRAS-CHAVE: SÍTIOS DE REDES SOCIAIS, FACEBOOK, PROSUMO

MOTIVATION, CONSUMPTION

AND FACEBOOK'S APPRAISALS

\begin{abstract}
Facebook has become the Social Network Site (SNS's) most popular in Chile. Thousands of young people who establish an identity on this site in order to build your own network of contacts and then generate socialization that directly affect both their privacy and their social-emotional life.

This communication aims to describe and analyze the motivations, uses and consumption made by users of the network UCSC Chile on Facebook.com conducted from a qualitative perspective.

The findings suggest that Facebook's written more than a social space is a cultural product whose consumption at the macro level has become fashionable. Despite the co-content tended to «prosumption», because the users are also producers in this environment that prioritizes strong and weak ties of personal communities.
\end{abstract}

KEY WORDS: SOCIAL NETWORK SITES, FACEBOOK, PROSUMPTION 


\section{INTRODUCCIÓN}

LA MASIFICACIÓN DE LOS Sitios de Redes Sociales (SRS), ${ }^{1}$ a partir de los años 2002-2003 ha constituido un creciente foco de interés para los estudios de comunicación mediada por computadora.

Para Ellison y Boyd (cf. 2008:210-230) los SRS corresponden a servicios basados en la web donde se les permite a los usuarios construir un perfil público o semi público, articular una lista de usuarios con los cuales comparten una conexión y, por último, ver y atravesar sus listas de conexiones que han establecido con otros usuarios de sistema.

Según los estudios de Haythornthwaite (cf. 2005:125-147) los SRS corresponden a formas diferentes de socialización, distintas de las que ocurren en las comunidades virtuales corrientes, pues en estas últimas no es necesario un vínculo de carácter offline. ${ }^{2}$ Tal como señala Mark Zuckerberg (Locke, 2005), fundador de Facebook.com, los SRS no se piensan como comunidades, ya que no intentan generar nuevos vínculos, sólo mantener los ya existentes en el mundo real.

De acuerdo al portal compete.com, Facebook se posiciona dentro de los cinco sitios online más visitados de la web y, además, es el número uno de los SRS; superando incluso a MySpace, portal que hasta el año 2008 lideraba el ranking en las listas de esta tipología de sitios (Kazeniac, 2009).

En Chile los SRS han alcanzado una gran popularidad mediante dos casos particulares: Fotolog y Facebook. Tanto así que en el primero Chile tiene la mayor cantidad de registrados a nivel mundial (Universidad Central, 2008).

En nuestro país, la empresa fundada por Zuckerberg alcanzó su mayor cantidad de registros durante febrero de 2008, cuando apareció la versión en español (Schek, 2008). Con esto nuestro país se ubicó en segundo lugar a nivel latinoamericano en cuanto a la cantidad de usuarios registrados (CADEM, 2008).

El estudio más importante realizado hasta la fecha en Chile sobre Facebook es de Cadem Research Internacional (2008) por encargo de la Corporación de Televisión de la Universidad Católica de Chile (Canal 13). Éste reveló importantes parámetros que sirven de pauta para

1 Social Network Sites, SNSS (según la tradición inglesa).

2 Las relaciones offline se refiere a aquellas que se establecen en la vida real, es decir, fuera de internet. 
comprender el consumo masivo y el comportamiento general de los usuarios en esta plataforma.

Los objetivos de esta investigación fueron caracterizar y analizar las motivaciones, usos y consumo que los usuarios miembros de la red UCSC Chile realizan en Facebook.com desde una perspectiva cualitativa.

\section{EXPLICACIONES METODOLÓGICAS}

Para el presente estudio se utilizó la técnica del focus group. La pertinencia de dicha técnica radica en explorar aspectos relevantes que determinan el consumo de la plataforma ${ }^{3}$ www.facebook.com, los cuales se manifiestan en los discursos de cada individuo.

\section{a) Participantes}

Se realizaron 3 grupos de discusión, los cuales estuvieron conformados por alumnos de ambos sexos de la Universidad Católica de la Santísima Concepción (UCSC) provenientes de distintas carreras: periodismo, historia, trabajo social, ingeniería civil en informática, ingeniería civil industrial, ingeniería comercial y medicina. Así también, como de diferentes grupos etarios, con edades que fluctuaron entre los 18 y 29 años.

La selección de los participantes se hizo mediante el conocimiento previo de los perfiles de la red UCSC Chile de Facebook, a los cuales se tuvo acceso al ser partícipes de dicha red. Dichos grupos focales se desarrollaron en dependencias de la UCSC, en la ciudad de Concepción, durante octubre de 2008.

\section{b) Instrumento}

El instrumento se confeccionó a partir de los siguientes tópicos: i) razones para unirse a Facebook; ii) agrado/desagrado frente a la plataforma; iii) motivación por pertenecer a la red UCSC Chile; iv) confianza en los demás usuarios de la red; v) modo de relación; y vi) actividades más comunes. Lo que se tradujo en un cuestionario con las siguientes preguntas: ¿por qué se unieron a Facebook?; ¿qué les agrada

3 Como señala Baquerín de Ricitelli (2007) la experiencia en entornos virtuales está íntimamente relacionada con las motivaciones iniciales que conllevaron la unión del usuario en la plataforma. 
y qué les molesta de Facebook?; ¿cuáles son los motivos por los que se han incorporado a la red UCSC Chile de Facebook y qué razones han evitado que se unan?; ¿creen o desconfían en los datos que se publican en los perfiles de Facebook?; ¿de qué modo se relacionan con los demás usuarios?; y ¿cuáles son las aplicaciones que más utilizan en el portal?

\section{c) Procedimiento}

En una primera instancia se citó a un grupo conformado por ambos géneros. La reunión duró 47 minutos y en la ocasión acudieron siete usuarios del portal (cuatro hombres y tres mujeres), de los cuales la gran mayoría (6 de 7) pertenecía a la red UCSC Chile.

Después de esta primera sesión quedó en evidencia que el pertenecer a la red UCSC Chile tenía mínima importancia, puesto que los asistentes señalaron desconocer la utilidad de pertenecer a ella.

En una segunda etapa la invitación se realizó sólo a mujeres, aquí participaron seis estudiantes. Cabe destacar que no todas sabían si realmente pertenecían o no a la red UCSC Chile. La duración de este grupo de discusión fue de 55 minutos.

Finalmente el tercer grupo estuvo conformado sólo por hombres, participaron 9 estudiantes. Duró 40 minutos y acá la mayor parte no dijo pertenecer a la Red UCSC Chile de Facebook.

Posteriormente, toda la información recabada fue transcrita íntegramente a papel. Luego fue seleccionada y fragmentada en 11 categorías, cada una de ellas respectivamente operacionalizada. Todo esto se tradujo en un informe que revela las respuestas a nuestras interrogantes a modo exploratorio.

\section{EXPOSICIÓN DE RESULTADOS}

\section{a) Motivos para unirse a Facebook}

El principal motivo reconocido por los estudiantes que participaron del estudio para unirse a esta plataforma fue la popularidad que había alcanzado Facebook en Chile, por lo que consideraron que era importante ser parte de esta nueva moda. Como lo grafica la siguiente sentencia: «Veía que mis compañeros se iban agregando y yo iba quedando fuera... así que decidí unirme». 
Si bien muchos de los participantes se habían negado en un principio a la idea de tener una cuenta en Facebook, ya que no les llamaba la atención o desconocían de qué se trataba, ahora afirmaban que: «es importante para estar en la onda».

Lo anterior, según la mayoría de los informantes, ha producido presión social, con tal de que más gente se una a este sitio. Algunas experiencias se grafican así: «Me llegaban como 'chorrocientas mil' invitaciones al Messenger, entonces me sacó de quicio».

En otros casos, fueron conocidos, compañeros o amigos los que incitaron, y a la vez ayudaron a crearles sus cuentas de usuario, como en la siguiente situación:

Cuando estaba haciendo la práctica todos tenían un perfil y yo no sabía qué era, entonces una compañera de allá me dijo: «si todos tenemos uno, ¡cómo no vas a tener!, entonces ella misma me creó la cuenta».

Se dio el caso de dos estudiantes que prácticamente se vieron obligados a crear su perfil en Facebook, ya que de seguir en la negativa simplemente no tenían acceso a ver cierta información, ya que sus amigos no se daban la molestia, por ejemplo, de enviar fotografías por e-mail. Ellos señalaron que: «cuando querían mandarme algo no podían, porque yo no tenía Facebook». Mientras el otro comentó: «un amigo por MSN me decía: «oye quiero que veas una foto», «ya poh mándamela», «no, es que está por Facebook», «ya y ¿cómo las veo?», «tienes que inscribirte», «es que no quiero» hasta que finalmente tuvo que hacerlo.

Otro de los motivos importantes para tener un perfil en el sitio fue saber que a través de Facebook podían volver a vincularse con personas que conocieron en el pasado. Comentaron que:

Uno logra encontrar gente que sin Facebook, nunca más la hubiese visto; [...] podías encontrar y ver a distintas personas sin tener mayores datos que su nombre, así podías reencontrarte con compañeros de curso, vecinos, amigos.

Esta revinculación y reafirmación en los lazos afectivos, aparentemente emociona a los usuarios:

Me reencontré con gente que no veía hace muchos años y eso me fue emocionando; [...] es interesante poder saber qué pasó con la vida de tantas personas que hace tanto tiempo habías dejado de ver, eso fue lindo. 
Para muchos de los participantes este reencuentro les sirvió además para incrementar su círculo social, ya que al tener una nueva forma de comunicarse se hacía más fácil volver a juntarse y rehacer amistades de infancia.

Por otra parte, las ventajas y múltiples aplicaciones que entrega Facebook es un gran atractivo para tener una cuenta. La gran mayoría de los participantes respondieron que era mucho mejor que los sitios que conocían hasta ahora y de forma natural establecieron comparaciones con el Fotolog y el MSN, que eran los medios que más usaban previamente para comunicarse, lo que queda demostrado con afirmaciones como las siguientes:

Es algo mucho más entretenido que el Fotolog y el MSN; [...] yo siempre estuve esperando una página distinta a Fotolog, que lo encuentro muy masivo.

\section{b) Agrado y desagrado en Facebook}

Según los participantes está claro que Facebook tiene la ventaja de contar con variados servicios en un solo lugar, a los informantes les agrada que: «En una misma página tienes el acceso de mandar un mail, postear, mandar mensajes», lo que sin duda simplifica la vida social de la que son parte.

En apariencia Facebook es un medio que ha ido desplazando a otras plataformas por ser considerada «más completa». A los participantes les gusta que: «Te recuerda los cumpleaños»; «puedes ver fotos de un viaje que hizo cierta persona»; "sirve como agenda»; «tiene juegos en línea»; «tiene chat»; «puedes subir videos». En síntesis, Facebook une todo en uno.

Otra de las grandes ventajas expresadas fue lo económico que resulta este medio, ya que Facebook, por ejemplo, avisa de los cumpleaños de sus amigos y ahí mismo aprovechan de saludarlo, pero además tienen aplicaciones para enviar regalos virtuales gratis. Quizá por ello, según expresó uno de los informantes: «Facebook está dejando de lado al teléfono celular».

Además, están las ventajas técnicas de la plataforma, ya que es considerado por los estudiantes como: «súper fácil de manejar»; «súper sencillo y las aplicaciones son muy variadas». Por otro lado, entrega las opciones de configuración para que se pueda mantener un control sobre la exposición de la vida privada: «Tú eliges qué mensa- 
jes te llegan y cuáles no, qué es lo que tú quieres que Facebook te avise»; «todo se puede configurar». Aunque esto sólo es bien aprovechado por quienes manejan con destreza la plataforma.

Aparentemente, Facebook modifica los vínculos personales y esto es visto como una ventaja, en el sentido que sirve para generar una mayor cercanía con quienes son parte de su red de amigos, sobre todo si tienen entre sus contactos - por ejemplo- a los profesores:

Ellos también tienen Facebook, entonces uno se siente más cercano a ellos, te escriben en el muro o te mandan mensajes, uno se entera de las relaciones que tienen y eso es como súper buena onda.

Esta misma cercanía también se puede dar con extraños, ya que a través de los grupos, ${ }^{4}$ a los cuales se integran, se produce una vinculación con desconocidos, pero con quienes comparten cosas comunes. Esto es considerado como: «Una forma original de poder conocer a otras personas y poder compartir experiencias».

Como contraparte también hay aspectos específicos de Facebook que molestan a los usuarios, pero son bastante menos que las ventajas reconocidas, por lo tanto no tienen para ellos el peso suficiente como para cerrar su cuenta.

El común de los participantes comparte una visión similar a la hora de señalar sus desventajas, entre las que destacan: «Es demasiado copuchento». Esta sentencia va en directa relación, a la inherente capacidad de Facebook en dar a conocer información ajena: «Me molesta el hecho de que Facebook te dice de todo, y no sólo a ti, sino también al resto de la personas... la intimidad y la privacidad se pierde».

Como consecuencia de lo anterior surge, de manera espontánea, el resquemor a los potenciales peligros de exponer datos personales:

Uno no sabe para qué están usando la información que uno pone; [...] hay gente que uno acepta porque lo conoce no más, entonces se entera de toda la vida en el perfil... eso puede ser peligroso.

Otra gran desventaja que fue reconocida por un importante número de participantes, es el tiempo que dedican diariamente a estar conectados en la plataforma: "Creo que la gran mayoría gasta mucho tiempo... pero son horas»; «me da lata de repente no hacer cosas que tengo que

4 Grupos virtuales con fines específicos creados por los mismos usuarios de Facebook con la finalidad de obtener adeptos en las redes de contactos. 
hacer por estar 'pegado' en un juego». Tanto hombres como mujeres reconocen que a la larga termina siendo un «vicio».

Es mayoritariamente aceptado que Facebook aún tiene falencias en cuanto a proteger la vida privada de las personas sobre todo en el ámbito de las fotos, ya que aseguran: «No falta el amigo que sube una foto y me etiqueta, pone mi nombre y al final todos la ven». A pesar de que existe la posibilidad de demandar la foto para que ésta sea bajada del portal, no lo hacen, porque es un trámite que se demora y en ese lapso ya todos la han visto.

Por otra parte, si bien Facebook es reconocida como una herramienta bien completa, a juicio de los participantes hay aplicaciones que necesitan ser perfeccionadas, como por ejemplo el chat: «El chat es limitado»; «yo sólo lo uso en casos extremos, prefiero el MSN».

Existe un consenso general, en cuanto a señalar que las principales desventajas, están centradas en la gran exposición de la vida privada, y aunque se tiene la posibilidad de no publicar alguna cosa, existe la tentación de hacerlo, ya que si los demás lo hacen, se hace necesario que también el propio usuario vaya publicando sus cosas, para hacer más interesante su perfil. En consecuencia, parece ser que la estructuración del yo o la imagen de ese yo se construye y reconstruye permanentemente a partir de toda la información que aparece en el perfil.

Conocer de manera más acabada la plataforma puede, paradojalmente, conllevar una gran molestia reconocida por los invitados, que es la saturación del e-mail, por los incontables avisos de Facebook:

No me gusta que me lleguen todas las notificaciones al correo, me carga, siempre tengo que estar borrándolas; [...] a veces llego a mi casa, abro mi mail, digo joh 20 mails! ojalá sea mi mamá o mi papá, no, es Facebook.

\section{c) Pertenecer a la red UCSC}

En general, los participantes señalaron desconocer la existencia de una red universitaria dentro del propio Facebook, y por lo mismo, difícilmente reconocerían eventuales aspectos positivos y/o utilidades, expresando que: «Sé que hay un asunto de UCSC y estoy, pero no tengo idea de qué es».

Otro aspecto relevante es que los alumnos que sí pertenecen, no encuentran en ello un real provecho o beneficio. Quienes están insertos lo hacen sólo porque estudian en esta casa de estudios: 
Pensé que podría llegar algo interesante a mis manos, pero ahora no encuentro que haya nada que valga la pena revisar, indagar e investigar. No vale la pena pertenecer.

Las opiniones relativamente positivas con respecto a ser parte de la red UCSC Chile, se orientan en el sentido de pertenencia e identificación con la institución:

Estoy por pertenecer a algo, porque en realidad todos mis compañeros se unieron a la red, estamos todos dentro de un grupo de estudio; [...] yo me metí para identificarme que también estudio acá.

La única ventaja que encuentran al pertenecer a esta red es el nivel de acceso que tienen a los perfiles de todos los usuarios que son parte de ella, ya que pueden ver la vida de gente que conocen, pero con la cual no tiene lazos de amistad. Aunque también hay usuarios que configuran el perfil y lo dejan como no visible.

\section{d) Socialización en Facebook}

Las formas de socializar en Facebook son variadas, pero también lo son los distintos niveles de cercanía que el usuario está dispuesto a desarrollar con la plataforma. Se dio solamente un caso - en el grupo de hombres- en que el usuario no destinaba el sitio para generar una red social: «No me relaciono con los usuarios del Facebook, creo que es un mal medio para hacerlo, es más para saber cosas».

Sin embargo, la gran mayoría considera que el gran atractivo de Facebook es la capacidad que tiene de acercar a las personas y establecer vínculos, como por ejemplo: «a través de una foto puedes descubrir ciertas cosas que a lo mejor pueden cambiar la percepción que tenías de alguien, me gusta eso de conocer más allá de lo que entrega una persona normalmente». Otra opinión fue que: «Computacionalmente es más fácil entablar una conversación con alguien que no conoces, y después hablar en persona».

Al preguntarles sobre las aplicaciones que utilizaban para socializar los participantes consideran que todo depende del grado de confianza que exista con los otros contactos, por ejemplo: «con mis amigos en Facebook tenemos una relación informal así que por lo general suelo escribirles al muro» ya que éste sólo es usado cuando quieren dejar un saludo que no sea comprometedor. 
Con respecto a las fotos y los comentarios se generó mayor polémica, ya que muchos consideran que es la aplicación más atractiva para los posteos. Sin embargo, también es una forma de vulnerar la intimidad personal de otros. Esto sucede en el momento en que un tercero, sube o publica una fotografía en donde aparece el propio usuario: "Antes de subirla, debería pedirle autorización a uno, para aceptar o no la foto, no falta el amigo que te etiqueta y al final todos te ven haciendo el ridículo». Esto los deja vulnerables a los comentarios de gente que ni siquiera conocen.

Otra de las formas de socializar, y que además permite involucrar a personas ajenas al círculo de amistad dentro del sistema, es la que se da a través de los grupos. Aplicación muy usada y que genera mayor deleite entre los usuarios, ya que ahí conocen gente que comparte sus mismos intereses. Dentro de las mayores motivaciones para pertenecer a un grupo está el entretenimiento y el sentido de pertenencia: «Yo pertenezco como a sesenta grupos»; «Uno ve el tema del grupo y según eso te unes y te vas comportando».

\section{e) Aplicaciones más utilizadas}

Al parecer el uso de las aplicaciones está íntimamente ligado a las fases o etapas por las que el usuario va pasando al servirse de la plataforma. Los usuarios reconocen que en un principio ocupan más El Muro, para postear o escribir a los amigos que encuentran: «Si me escriben en el muro voy a escribir en el muro de la persona que me escribió». En otros casos, el hecho de que sea visible relega esta aplicación sólo para mandar mensajes que no sean comprometedores: «lo uso más que nada con la familia, hacerle burla a los primos, ese tipo de cosas».

Subir fotos y comentarlas también está dentro de la iniciación en Facebook, pero parece ser que a medida que superan la barrera de la mera curiosidad inicial, la herramienta se vuelve más útil y se diversifica su uso, por ejemplo, en la utilización de notas donde se espera «que se genere alguna especie de debate, donde la gente comente lo que piensa».

Otra aplicación considerada muy provechosa, es la agenda de eventos, como ayuda memoria: "Con una semana de anticipación te avisa los eventos de la semana y todos los que están de cumpleaños, y aprovechas ahí mismo el medio para decir, ;feliz cumpleaños!».

Facebook es considerado por los usuarios como un medio que sirve máxime como entretención y es por eso que son variadas las aplicaciones que están destinadas a eso, como la música, los juegos y 
los test: «Los juegos son bien interesantes porque uno va compitiendo con los amigos en la vida real, y el hecho de que se publique un fichero con un ranking, te hace estar más metido para ir ganándole a tus amigos». Si bien los usuarios no encuentran complejos los juegos, reconocen que se han transformado en un vicio, porque en muchos de ellos está en juego la imagen, al ser públicos sus resultados.

En el caso de los test, la opinión general es que su finalidad es: «resaltar la personalidad». En un comienzo, la mayor parte de los usuarios respondía una importante cantidad de ellos. Sin embargo, al pasar el tiempo, éstos sólo se hacen cuando no hay nada más interesante que hacer.

\section{f) Confianza en los demás usuarios de la red}

En apariencia la mayoría de los usuarios confían en los perfiles que ve publicados y en las historias que lee en los muros, pues: «son personas de confianza, que uno conoce». Así mismo aseguró otra participante: «Por lo general son los amigos y uno tiende a conocer la gran mayoría de los perfiles».

Sin embargo, los estudiantes reconocieron que entre sus contactos hay varios extraños o desconocidos, y tienen sus reparos al respecto: «Hay que desconfiar más de las invitaciones que llegan», sentencian. La exposición de la vida privada es enorme y prácticamente no existe forma de enterarse en qué se usará la información que muchas personas hacen pública, por el hecho imperioso de estar ubicables:

Yo sé que es una estupidez, pero en mi caso tengo toda la información publicada, mi teléfono, mi mail, eliminé todas las opciones de seguridad.

«La gente tiene una capacidad tremenda para engañar a otras personas». A pesar de esta última sentencia, hasta el momento ninguna persona reconoce haber tenido alguna mala experiencia en Facebook: «No me ha tocado ese tipo de cosas».

Sí reconocen que la omisión o engaño se da más usualmente en el estado civil, ya que éste es un medio muy utilizado para conocer gente y flirtear, los participantes aseguran que mucha gente: «No comenta lo que no les conviene». A pesar de ese hecho tampoco consideran que sea una falta grave: «No te está mintiendo en grandes cosas».

En este ítem surgió la comparación de seguridad que entregan otras plataformas muy utilizadas como el MSN y Fotolog. La conclusión gene- 
ral fue que todas son vulnerables: «Han falseado Fotolog, o han creado Fotolog con otras identidades para saber qué pasó con tal persona», y en el caso de Facebook, por lo general, dicen que no hay forma de saber quiénes realmente han visitado tu sitio para obtener datos.

\section{g) Diferencias de percepciones entre hombre y mujeres}

Las posibles dicotomías entre géneros, no están en los tiempos de uso ya que como aseguran los informantes «Los hombres y las mujeres están igual de 'pegados' con Facebook». La diferencia radica en las aplicaciones que utilizan, ya que las mujeres consideran que los hombres son más mecánicos y que por eso «ocupan más los juegos» mientras que ellos piensan que las mujeres son más sensibles e inseguras por lo que «usan los test para reafirmar su autoestima».

Las mujeres reconocen que usan Facebook más que nada para mantener lazos de comunicación y enterarse de la vida de la gente. Es por esto que a la hora de ingresar a su perfil se dedican más a: «Comentar lo que están haciendo y subir las fotos de los eventos que surgen», pero no para: «Estar haciendo guerra de pandillas ni jugando». A diferencia de los hombres que ven en la plataforma un medio netamente de entretención. Es por eso que destinan su tiempo de uso en: «Jugar»; «hacer grupos, molestar a sus compañeros». La gran mayoría de ellos no se comunican a través de las aplicaciones que existen: «Encuentran que es un mal medio para hacerlo»; «es más para saber cosas».

\section{DISCUSIÓN}

\section{a) Análisis teórico}

Al analizar los motivos para unirse a esta plataforma podemos decir que existe congruencia con los postulados de la Escuela de Frankfurt. Siguiendo a Adorno y Horkheimer, el entorno de Facebook.com supone un producto o un «servicio» desarrollado por las industrias culturales, que masifican y homogeneizan las prácticas de consumo, pero éstas sólo pueden ser entendidas como tales desde una perspectiva macro, pues la emergencia del prosumidor (cf. Urresti, 2008:53-56) hace que todos los productos se centren en la mayoría de los casos, en individuos cercanos a nivel físico para los consumidores activos.

Como resultado, el proceso de integración a este sitio se convierte en moda, esto se ve reflejado en afirmaciones como las siguientes: «La 
mayoría llegamos de 'monos', porque uno ve al vecino responder algún test o jugar algún juego y luego lo imitamos».

Podemos distinguir entre usuarios que sabían para qué servía este sitio antes de registrarse y los que no. Los primeros corresponden a personas que ingresaron con una finalidad específica, mientras que los segundos se adhieren al website, por curiosidad y moda.

Los motivos de los informantes que declararon haber tenido nociones de Facebook antes de integrarse se relacionan con lo que Ellison, Lampe y Steinfield (2007) sondearon en la Michigan State University: resucitar relaciones del pasado. Esto queda graficado en la siguiente frase: «supe que Facebook unía compañeros de generaciones anteriores y quería generar una unión con ellos nuevamente».

Por otro lado, para algunos fueron «los amigos y amistades cercanas» quienes los llevaron a conocer Facebook. Esto tiene su fundamento en lo que Caroline Haythornthwaite (2005:125-147) denomina conectividad entre los lazos fuertes y lazos débiles, que son los que van a propiciar la entrada a una red, pues los lazos latentes e inexistentes no representan un interés particular.

Los estudios del comportamiento del consumidor considerarían la integración a Facebook como una decisión racional que evalúa los costos y beneficios de unirse al sistema, pues Facebook representa la unión de múltiples servicios: «Uno tiene el acceso a la mano de mandar un mail, postear, mandar mensajes», además «tiene juegos». En consecuencia, «une todo en uno».

Como ya se ha adelantado en lo que a comportamiento de consumidor se refiere, Facebook tiene la ventaja de ser una plataforma que «integra servicios muy variados». Además, sirve para «mantener el contacto» $\mathrm{y}$ 《estar actualizado» de historias locales, que acontecen a personas cercanas y que son narradas a través de fotografías digitales y comentarios fragmentados en posteos, ${ }^{5}$ evidencias de la estética postmoderna.

Como sugiere Morley, la posmodernidad corresponde a una nueva sensibilidad cultural que involucra un estilo estético (Morley, 1998:85), vinculado a la hiperrealidad, en la que lo importante ya no es la realidad, sino la simulación de la misma (Baudrillard, 1978:14). Los usuarios simulan la realidad por medio de «la selección de las fotos que suben» o «no colocan el estado de relaciones» y crean su propia realidad a través de «comentarios en los otros muros».

5 Comentario ingresado por un usuario a un perfil. 


\section{b) Conclusiones}

De acuerdo a los resultados obtenidos en los grupos de discusión efectuados en el marco de este estudio, podemos señalar las siguientes respuestas a las preguntas de investigación.

En el caso de las mujeres parece ser que el principal motivo para unirse a Facebook es la presión del grupo y la necesidad de mantener lazos afectivos. En el caso de los hombres, en cambio, la diversión o entretención prima por sobre los aspectos socializadores.

Como segundo tópico, la credibilidad que los usuarios atribuyen a los perfiles que exploran parece ser determinada por la cercanía con el autor de dicho perfil, pues en la mayoría de los casos y como efecto de la nominalidad real alcanzada en Facebook los usuarios, generalmente, saben con quien están interactuando.

Como tercer punto cabe señalar que las formas de socialización en Facebook están determinadas por el grado de confianza que exista con los otros contactos y sobre esa base utilizan algunas de las herramientas de la plataforma para hacerlo. Cuando la relación es informal usan «El Muro para saludar», en el caso de las mujeres, y los comentarios de fotos. Sin embargo los hombres socializan más a través de «los grupos» que son de carácter lúdico, donde no se exige un mayor compromiso social.

Un cuarto aspecto referido a las aplicaciones más usadas considera que el uso de las herramientas o recursos que Facebook entrega, está marcado por una diferencia de género. Las mujeres utilizan más todos los recursos, que desde la perspectiva de Naomi Baron son denominados como interacciones online, los cuales corresponden a herramientas como el muro, mensaje personal y el toque. Esto debido a que el uso que le dan a su perfil está direccionado hacia mantener lazos comunicacionales.

A diferencia de ellas, pareciera ser que los hombres encuentran en Facebook un espacio que está más destinado al ocio que a mantener vínculos sociales. De esta forma, sus preferencias se inclinan hacia los juegos y algunos test de carácter masculino.

De los focus group se infiere que la coexistencia que involucra el proceso de mediamorfosis propuesto por Fidler (1997) se está dando en torno a herramientas que yacen en los sitios web. En otras palabras, la aparición de Facebook no supone la eliminación de MSN ni de Fotolog, sino por el contrario, la complementariedad hace que las herramientas entre sí mejoren. 
Según la información recolectada parece haber evidencia que la apropiación efectiva de las nuevas tecnologías de la información acontece de forma limitada en los grupos de informantes que participaron en las sesiones, pues si bien todos tenían entre 18 y 29 años sólo uno de los 22 participantes se autodenominó como usuario avanzado. Por último, es preciso remarcar que todos los participantes eran estudiantes universitarios, por lo que compartían cierto capital cultural y simbólico.

CONCEPCIÓN (CHILE), AGOSTO 2009

RECIBIDO: SEPTIEMBRE 2009

ACEPTADO: OCTUBRE 2009

\section{REFERENCIAS BIBLIOGRÁFICAS}

BAQUERIN DE RICITELli, MARÍA TERESA (2007): Internet: motivación y conocimiento. Estudio cualitativo de la intencionalidad de los usuarios. Buenos Aires: Educa.

BAUDRILLARD, JEAN (1978): Cultura y simulacro. Barcelona: Editorial Kairos.

CADEM RESEARCH INTERNATIONAL (2008): «Facebook fenómeno en Chile». Disponible en http://cademresearchinternational.files.wordpress.com.

Ellison, Nicole; ChaRles STEINFIELD y CliFF LAMPE (2007): «The benefits of Facebook 'friends': Social Capital and College Students' Use of Online Social Network Sites». Journal of Computer-Mediated Communication $\mathrm{N}^{\circ} 12$ (4).

— y DANAH BOYD (2008): «Social Network Sites: Definition, History, and Scholarship». Journal of Computer-Mediated Communication $\mathrm{N}^{\circ} 13$.

FIDLER, ROGER (1997): Mediamorphosis: understanding new media. California: Pine Forge Press.

HAYThornthwaite, CARoline (2005): «Social Networks and Internet connectivity effects». Information, Communication, \& Society $\mathrm{N}^{\circ} 8$ (2).

HORKHEIMER, MAX y THEODOR ADORNO (1998): Dialéctica de la ilustración. Madrid: Trotta (tercera edición).

KAZENIAC, ANDY (2009): «Social Networks: Facebook Takes Over Top Spot, Twitter Climbs». Disponible en http://blog.compete.com.

LOCKE, LAURA (2007): «The future of Facebook». Time, 17 de julio de 2007. MORLEY, DAVID (1998): «El posmodernismo: una guía básica». En CURRAN, MORLEY y WALKERDINE: Estudios culturales y comunicación: análisis, producción y consumo cultural de las políticas de identidad y el posmodernismo. Barcelona: Paidós. 
URRESTI, MARCELO (2008): «Ciberculturas juveniles: vida cotidiana, subjetividad y pertenencia entre los jóvenes ante el impacto de las nuevas tecnologías de la comunicación y la información». En MARCELO URRESTI (editor): Ciberculturas juveniles: los jóvenes, sus prácticas y sus representaciones en la era de internet. Buenos Aires: La Crujía.

SCHEK, ALEXANDER (2008): «Chile es el país de mayor crecimiento en Facebook a nivel mundial». Disponible en www.fayerwayer.com. 